\title{
Guillain-Barré Syndrome
}

\author{
Alejandro A. Rabinstein*
}

Division of Critical Care Neurology, Department of Neurology, Mayo Clinic College of Medicine, Rochester, MN, USA

\begin{abstract}
Guillain-Barré syndrome (GBS) is caused by an inflammatory polyradiculoneuropathy, which most commonly results from acute demyelination produced by a CD4 T-cell mediated response against myelin proteins. Axonal forms have also been recognized. Molecular mimicry between components of the bacterial wall of Campylobacter jejuni and gangliocytes in the membranes of peripheral axons may be responsible for some cases of axonal GBS. Immune therapy with plasma exchange or intravenous immunoglobulin is the standard of care for the treatment of patients with acute GBS. This review summarizes available information regarding the pathophysiology, clinical manifestations, therapeutic considerations, and prognosis of this disorder.
\end{abstract}

Guillain-Barré syndrome (GBS, or better Guillain-BarréStrohl syndrome to recognize the three authors responsible for the first description of this disorder) [1] is an inflammatory polyradiculoneuropathy characterized by the acute development of diffuse weakness and areflexia. This syndrome encompasses various subtypes, the most common of which is acute inflammatory demyelinating polyradiculoneuropathy (AIDP). Less common are the axonal variants, acute motor axonal neuropathy (AMAN) and acute motor and sensory axonal neuropathy (AMSAN), and the atypical forms with more localized clinical expressions such as the Fisher syndrome [2]. AIDP is responsible for most cases of GBS in the United States and Europe [3,4]. Axonal forms are more common in China, Japan, and perhaps Central America [5,6].

\section{EPIDEMIOLOGY}

Most population-based studies report an incidence of 1.2 and 1.9 cases of GBS in 100,000 individuals per year $[2,4]$. The incidence tends to increase steadily with age. Men are affected slightly more often than women. Most cases are sporadic and exceed a relatively stable incidence in the population over the years. However, epidemic outbreaks of cases of GBS have been reported, most notably, summer outbreaks in China linked to contamination of water by Campylobacter jejuni $[5,7]$.

Preceding infections are noted in nearly two-thirds of patients with GBS $[8,9]$. Implicated microorganisms include Campylobacter jejuni, cytomegalovirus, Epstein-Barr virus, Haemophilus influenza and Mycoplasma pneumoniae. Immunizations with vaccines have been linked with recurrence of GBS. Over the last two decades there has been a steady decline in the number of cases of GBS related to influenza vaccine. The risk of developing GBS as a complication of influenza of vaccine at the present time is no larger than 1: $1,000,000$ [10]. Rabies vaccine containing brain material may result in GBS in 1: 1,000 immunizations. There is no convincing evidence that any other vaccines carry a risk of

*Address correspondence to this author at the Mayo Clinic, Department of Neurology, W8B, 200 First Street SW, Rochester, MN 55905, USA; Tel: 507-538-1036; Fax: 507-266-4419; E-mail: rabinstein.alejandro@ mayo.edu inducing GBS as a complication. Sporadic cases of GBS can occur after surgeries, in patients with cancer, after transplantations, and in patients with HIV infection [11]. The rather paradoxical occurrence of GBS, an autoimmune condition, in patients with immune depression is likely explained by a dysregulation of the mechanisms modulating immune responses.

\section{PATHOPHYSIOLOGY}

The pathogenesis of GBS varies across subtypes. In AIDP the predominant mechanism responsible for the demyelination seems to be a CD4 T-cell mediated response against myelin proteins (P2, P0, or PMP22) [12]. Multifocal mononuclear cell infiltration is observed in peripheral nerves, nerve root, and cranial nerves throughout the body. Myelin destruction is caused by invasion of macrophages targeted against antigens on the membrane of Schwann cells or the myelin sheath [13]. Activation of macrophages is mediated by $\mathrm{T}$ lymphocytes. An alternative pathophysiological theory implicates a complement-mediated myelin attack [14]. Axons are not the primary immunological target in this form of the syndrome. No antibodies have been found to have a pathogenic role in the genesis of AIDP.

The axonal forms of GBS, such as AMAN, have a different pathophysiology. The lack of lymphocytic infiltrates in pathological samples attest to the lack of significant activation of cellular immunity. Instead, antibodies directed against specific antigens on the axolemma are responsible for the activation of macrophages that then attack the nodes of Ranvier leading to axonal damage with relative preservation of the myelin sheath [15]. Cases of AMAN often have detectable antibodies against gangliosides in the serum. Different subtypes of axonal GBS are associated with different antibodies, although the associations are far from exclusive. The most frequently found antibodies are GM1 and GM1b in patients with AMAN, GD1a antibodies in patients with acute sensory neuronopathy, and GQ1b antibodies in patients with the Fisher variant (although this seems to be more a demyelinating than an axonal disorder) [16,17].

Molecular mimicry between components of the bacterial wall of Campylobacter jejuni and gangliocytes in the membranes of peripheral axons is thought to be responsible for 
the cases of axonal GBS triggered by Campylobacter jejuni infection [17-19]. Lipo-oligosaccharide (LOS) is one of the most important cell-surface structures expressed by Campylobacter jejuni [20]. The terminal tetrasaccharides of the purified LOS have been shown to be identical to those encountered in GM1 ganglioside [21]. Genetic studies assessing the effect of truncated forms of LOS on antibody formation indicate that a certain core oliogosaccharide (HB93-13) appears to be essential for the induction of anti-GM1 (and anti-GD1a) antibodies [22]. In an experimental model, Japanese white rabbits repeatedly injected with an LOS fraction of Campylobacter jejuni containing the GM1-like portion were found to develop pathological and immunohistochemical changes compatible with the features of human AMAN [23]. Infection with Campylobacter jejuni bearing a GT1alike or GD1c-like LOS could also induce anti-GQ1b IgG antibodies in patients with the Fisher variant [20]. There is no evidence that this mechanism of molecular mimicry triggered by Campylobacter jejuni infection can induce AIDP [24].

Similar mechanisms of a cross reactivity between microbial and nerve antigens have been postulated for other microorganisms (Haemophilus influenza, Mycopplasma pneumoniae, and cytomegalovirus) that may result in cases of AMAN and Fisher variant, but the supportive evidence is much weaker than in the case of Campylobacter jejuni [20]. It is not clear why only certain patients exposed to these infections develop a complication in the peripheral nervous system since no specific immunogenetic markers have been identified in populations of patients with GBS.

\section{CLINICAL PRESENTATION}

The clinical presentation of GBS is often quite characteristic but far from uniform. The first manifestations are typically sensory symptoms. Dysesthesias are reported in the distal portions of the limbs and tend to be symmetric. Often patients complain of pain that may involve the lower back or the limbs. Weakness only begins to develop within the next one to two days. The speed of progression of the weakness is a good predictive marker of the subsequent severity of the disease. Hence, the risk of developing severe muscle paralysis and possible respiratory failure correlates with the rate of progression of weakness.

Over the course of the disease, hyporeflexia or areflexia always become apparent. However, initially patients may have normal reflexes or even be hyperreflexic. In fact, the presence of hyperreflexia in the very early stages of GBS is not at all uncommon and may be related to the disruption of inhibitory mechanisms at the level of the spinal cord [25]. The muscle weakness tends to involve proximal muscles early and tends to affect the legs earlier than the arms. The denomination of ascending paralysis is therefore related to the sequence of leg followed by arm involvement rather than a distal to proximal progression. The severity of weakness characteristically reaches its peak in less than four weeks [26]. The condition is classified as subacute if the peak of the severity of weakness is reached between four and eight weeks after symptom onset and chronic if such peak is reached more than eight weeks later.

Bilateral facial weakness if often encountered. Ophthalmoplegia is observed less frequently but it is a characteristic feature of the Fisher variant which also exhibits various degrees of ataxia along with generalized areflexia. Oropharyngeal weakness may occur, particularly in patients with marked bilateral facial weakness and ophthalmoplegia. It may result in dysphagia and, in most severe cases, also dysarthria. Risk of aspiration is increased in these patients. Bilateral ptosis is also a frequent feature in patients with ophthalmoplegia.

Neuromuscular respiratory failure is the most feared complication of GBS [27]. It occurs in up to $25 \%$ of patients. Diaphragmatic failure is primarily responsible for the ventilatory insufficiency and its cardinal clinical expression is the paradoxical breathing pattern. The degree of diaphragmatic weakness often correlates fairly well with the strength of neck flexion. As noted in the next section, bedside pulmonary function tests can be used to monitor, respiratory muscle function.

Autonomic disturbances represent another cardinal feature of GBS patients. Manifestations of autonomic dysregulation include postural hypotension, diaphoresis, tachycardia, ileus, bladder retention, hypertension, and potentially lifethreatening arrhythmias or cardiac arrhythmias [28-30]. Myocardial stunning has been reported with severe GBS [31]. Signs of autonomic dysfunction may dangerously worsen as the patient rapidly evolves into a respiratory failure.

Although primarily a motor disorder once the disease has been established, patients with severe GBS often complain of excruciating pain. Dysesthesias may also be prominent and quite refractory to treatment. The pain is experienced as deep and frequently has neuropathic features. It may be localized or generalized.

The differential diagnoses to be considered in patients presenting with acute paralysis is listed in Table $\mathbf{1}$.

\section{LABORATORY EVALUATION}

Electrophysiology represents the most important laboratory study to confirm the diagnosis of GBS in all its forms. Electrophysiological testing must be done as early as possible after presentation and should be repeated on a weekly basis to further confirm diagnosis and for prognostic purposes. Slow motor nerve conduction velocity is an early and characteristic finding of patients with AIDP [32]. Conduction blocks (drop in the muscle action potential amplitude when a site of proximal simulation is compared to distal stimulation of the same nerve) appear later, but are more specific of this disorder [32]. The sensitivity of electrodiagnosis is increased by testing $\mathrm{F}$ ways and $\mathrm{H}$ reflexes (which assess the integrity of the roots), both of which become abnormal early in the disease course. Sensory conduction is typically normal in the sural nerve and abnormal in the median nerve.

Another important aspect of studying the nerves early is that it provides the best chance to allow differentiation between demyelinating and axonal forms of GBS. In severe cases of demyelinating GBS, axonal loss will occur over time and, as it does, nerves become inexcitable. Presence of early motor nerve inexcitability represents a reliable marker of axonal polyradiculoneuropathy. 
Table 1. Differential Diagnosis of Patients with Acute Paralysis

\begin{tabular}{|l|}
\hline Brainstem stroke (ischemia, hemorrhage) \\
\hline Spinal cord compression \\
\hline Transverse myelitis (e.g. idiopathic, SLE, MS, WNV) \\
\hline Poliomyelitis \\
\hline Carcinomatous or lymphomatous meningitis \\
\hline $\begin{array}{l}\text { Infectious polyradiculoneuropathy (e.g. CMV in HIV-infected patients, } \\
\text { Lyme) }\end{array}$ \\
\hline Vasculitic neuropathy \\
\hline Acute intermittent porphyria \\
\hline Tick paralysis \\
\hline Poisonous bites (e.g. black widow spider) \\
\hline Organophosphate poisoning \\
\hline Ciguatera poisoning \\
\hline Critical illness myopolyneuropathy \\
\hline Myasthenia gravis \\
\hline $\begin{array}{l}\text { Persistent pharmacological paralysis (from neuromuscular blocking } \\
\text { agents) }\end{array}$ \\
\hline Botulism \\
\hline Periodic paralysis \\
\hline Severe polymyositis \\
\hline Hyperalimentation-induced hypophosphatemia \\
\hline CMV, cytomegalovirus; HIV, human immunodeficiency virus; MS, multiple sclerosis; \\
SLE, systemic lupus erythematosus; WNV, West Nile virus. \\
\hline
\end{tabular}

Lumbar puncture is also useful test in patients with GBS. The typical finding is elevation of spinal fluid protein level without any similar inflammatory response (albuminocytological dissociation) [1]. However, it is important to remember that the elevation in the protein level may not occur until days after the first symptoms of the disease have become present. Moreover, treatment with intravenous immunoglobulin may result in subclinical aseptic meningitis that may confound the cerebrospinal fluid profile by producing a rise in the number of nucleated cells.

Other diagnostic studies are mostly focused on excluding coexisting conditions, such as HIV infection and West Nile virus infection. Testing for ganglioside antibodies or markers of microorganisms that have been implicated in the pathophysiology of GBS has no proven practical value.

\section{GENERAL MANAGEMENT IN THE INTENSIVE CARE UNIT}

The most severe patients with GBS will require attention in an intensive care unit (ICU) setting. Admission to the ICU may be required for closer monitoring or treatment of complications of the disease. A list of potential indications for ICU admission is found in Table $\mathbf{2}$. The priorities of general ICU care in patients with GBS include management of respiratory failure, evaluation and treatment of manifestations of autonomic dysfunction, proper nutrition, pain control, and
Table 2. Indications for ICU Admission in Patients with Guillain-Barré Syndrome

\begin{tabular}{|l|}
\hline Neuromuscular respiratory failure \\
\hline Severe bulbar weakness with poor airway protection \\
\hline Aspiration pneumonia \\
\hline Marked blood pressure fluctuations \\
\hline Cardiac arrhythmias \\
\hline Rapidly progressive weakness \\
\hline Rapidly declining bedside respiratory parameters \\
\hline Sepsis \\
\hline Suspicion of pulmonary embolism \\
\hline
\end{tabular}

prevention of systemic complications such as infections and deep venous thrombosis [27].

Adequate care of patients with GBS in the ICU requires a multidisciplinary approach and must involve nursing staff with experience in the care of neurological patients, respiratory therapists with experience with patients with neuromuscular weakness, pharmacists, nutritionists, and physical and occupational therapists. Incorporating these various members of the team from the time the patient is admitted to the ICU is essential to maximize the quality of care.

\section{RESPIRATORY FAILURE}

The most common cause of respiratory failure in patients with GBS is progression of neuromuscular weakness leading to insufficient ventilation [33]. Inefficient inspiration is due to diaphragmatic weakness and results in alveolar hypoventilation. Therefore, the earliest expression of this disorder on arterial blood gases is a mild decline in $\mathrm{PO}_{2}$. This translates clinically into progressive recruitment of accessory respiratory muscles, tachypnea and dyspnea. Expiratory muscle weakness decreases the strength of the cough reflex, thus impairing the clearance of bronchial and tracheal secretions and increasing the risk of aspiration and pneumonia.

Bulbar weakness may compromise the ability of the patient to protect the airway [33]. Decreased muscle tone in the tongue and oropharyngeal muscles may predispose to mechanical airway obstruction particularly at night, when muscle tone physiologically decreases. Meanwhile, weakness of oropharyngeal, lingual and facial muscles may interfere with the normal protective reflexes (e.g. cough) and facilitate the occurrence of aspiration.

Patients admitted to the ICU with GBS should be examined at frequent intervals to detect any progression of neuromuscular failure. Useful clinical signs include respiratory rate, cough strength, span of numbers that the patient can count with a single breath, strength of bulbar muscles, strength of neck flexion and shoulder shrug, and, most noticeably, breathing pattern [34]. As mentioned earlier, paradoxical breathing is a marker of diaphragmatic failure and impending respiratory collapse [34]. It consists of the paradoxical inward movement of the abdominal wall muscles during inspiration. It is typically associated with significant respiratory distress manifested in the form of diaphoresis and 
tachycardia. By the time paradoxical breathing occurs, development of hypercapnia and profound hypoxia may follow quite rapidly.

Measurements of vital capacity and forced inspiratory and expiratory pressures can be performed at the bedside and are useful in monitoring patients with GBS. We typically monitor these parameters every four hours while the patient is awake and every six hours during sleep time. Traditionally, a forced vital capacity lower than $15 \mathrm{ml} / \mathrm{kg}$ is considered an indication for intubation and mechanical ventilation. However, in some instances this criterion may be rather conservative. In a study of 114 consecutive patients with severe GBS admitted to our Neurological ICU, we found that a vital capacity lower than $20 \mathrm{ml} / \mathrm{kg}$, maximal inspiratory pressure lower than $30 \mathrm{~cm} \mathrm{H}_{2} 0$, maximal expiratory pressure lower than $40 \mathrm{~cm} \mathrm{H}_{2} \mathrm{O}$, or a reduction of more than $30 \%$ in vital capacity, maximal inspiratory pressure, or maximal expiratory pressure were predictive of progression to mechanical ventilation [35].

It is important not to rely on any single factor when deciding whether the patient requires mechanical ventilation. This advice is particularly applicable to bedside respiratory measures because they are effort dependent and may be negatively influenced by bulbar weakness, which may result in insufficient sealing of the mouth around the spirometer. Thus, it is always safer to repeat the testing three times and use the best performance of the patient as guidance. On the other hand, it is always safer to intubate patients electively before a crisis ensues since emergency intubation in patients with GBS can have devastating consequences [36]. Apart from the respiratory function parameters mentioned before, rapid progression of weakness, bulbar dysfunction and bilateral facial weakness and signs of autonomic dysfunction are additional factors that predict requirement of mechanical ventilation (Fig. 1) [35]. Therefore, one should have a lower threshold to electively intubate patients that meet these criteria.

When evaluating arterial blood gases in patients with GBS, it is important to recognize that hypercapnia is a late phenomenon. Mild hypoxia initially occurs as a consequence of microatelectasis. Microatelectases are caused by diaphragmatic weakness. Patients in supine position tend to develop atelectasis in dependent lung regions which are also the areas receiving most blood flow in this position. The situation creates a ventilation- perfusion mismatch responsible for the mild hypoxia. Nonetheless, this situation tends to be mild in the initial phases of neuromuscular respiratory insufficiency. During this time, oxygen saturation may remain within normal range and the initial evidence of hypoxia may be erroneously dismissed as insignificant. Techniques of lung expansion in patients with marginal tidal volumes, such as frequent incentive spirometry and chest physiotherapy, may be of help at this time.

Signs of progression of respiratory muscle weakness, worsening of bedside respiratory function parameters, or persistent decline in oxygen saturation should be considered indications for elective intubation. Unlike what we have seen in patients with myasthenic crises, non-invasive ventilation

Fig. (1). Proposed algorithm for the management of patients with Guillain-Barré syndrome based on clinical and bedside respiratory parameters. VC, (forced) vital capacity; PImax, maximal inspiratory pressure; PEmax, maximal expiratory pressure. Modified with permission from Lawn et al. [35]. 
with BiPAP mask does not appear to be an effective alternative in patients with GBS [37]. Once the respiratory muscle weakness reaches a critical point, hypercapnia develops rapidly and is soon followed by profound hypoxia requiring emergency intubation. Sudden development of hypercapnia may also be caused by aspiration, mucous plugging, or upper airway obstruction from profound bulbar muscle weakness.

Once the patient is intubated, mechanical ventilation should be started. The most common ventilatory mode used in patients with GBS is synchronized intermittent mandatory ventilation (SIMV). Sometimes, at the very onset of mechanical ventilation, some patients feel more comfortable on assist-control mode. It is important for patients with GBS, especially those who required emergency intubation, to rest for the first 24 to 48 hours. To achieve this objective, the patient's work of breathing should be initially minimized by providing full mechanical ventilatory support. Ventilatory settings should include adequate levels of pressure support, positive end-expiratory pressure (PEEP) to prevent further atelectasis, and tidal volumes sufficient to achieve adequate lung expansion (low tidal volumes should be reserved for patients with pre-existent lung disease or evidence of acute lung injury). We typically use tidal volumes between 8 and $12 \mathrm{ml} / \mathrm{kg}$ in patients with neuromuscular respiratory failure such as GBS.

Post-intubation bedside respiratory tests can provide a measure to monitor respiratory status in GBS patients. An integrated pulmonary function score (PF ratio) has been described to guide the timing of extubation [38]. Progressive improvement in vital capacity and maximal inspiratory and expiratory pressures predict successful weaning from mechanical ventilation. It is useful to conceptualize the process of weaning as a form of respiratory reconditioning [27]. By doing so, one can easily understand why a period of high respiratory demand during which the patient does most of the effort should be alternated with adequate periods of rest, which in the beginning phases of weaning should span through the night. Weaning is achieved first by reducing the rate on the SIMV mode and subsequently by reducing pressure support. The practice of using T-piece trials is acceptable in patients without significant comorbidities and no evidence of active dysautonomia. However, these trials may not be tolerated by older patients and may be dangerous in patients with cardiac arrhythmia from autonomic dysregulation. Anxiety may play a significant role during the weaning process, especially in patients who have been intubated for longer periods of time and those who were emergently intubated because of severe respiratory distress. Verbal reassurance and, in some cases, prescribing a low dose of a minor tranquillizer such as alprazolam may be useful interventions.

In some patients with GBS, weaning may not be possible. Recognizing the need for tracheostomy may be important since early tracheostomy may reduce the incidence of complications from prolonged endotracheal intubation. Among other advantages, tracheostomy allows better suctioning of airway secretions and pulmonary toilet. Tracheostomies are most often needed in patients who are elderly or have preexistent pulmonary disease [39]. Lack of improvement of bedside respiratory parameters is also associated with increased chances of weaning failure and a need for a tracheostomy.
Pulmonary complications are the most common systemic complications in patients with severe GBS admitted to the ICU [40]. They include tracheobronchitis, pneumonia, and lobar atelectasis. Therefore, aggressive pulmonary toilet is mandatory; it should include frequent endotracheal suctioning using aseptic technique. Chest radiographs should be checked daily or every other day. Changes in the quality or quantity of respiratory secretions, leukocytosis and fever should be considered suspicious for pneumonia. After obtaining adequate respiratory samples for culture, it is reasonable to initiate antibiotic coverage for nosocomial pneumonia that may be stopped after three days if the diagnosis is not confirmed. Coverage for aspiration should always be considered in patients with GBS. The proper position of feeding tubes should be carefully confirmed prior to initiating enteral feedings since patients with GBS have impaired ability to protect themselves from aspiration.

\section{AUTONOMIC DYSFUNCTION}

Patients with severe GBS may present with the various complications from autonomic dysfunction including potentially fatal cardiac arrhythmias [41,42]. Hence, it is essential to keep patients with severe GBS under cardiac telemetry surveillance. While hypertension predominates, spells of hypotension may occur and are often unpredictable. They may be triggered by tracheal suctioning, bladder manipulation or ocular pressure. Apart from these vagal spells, hypotension can also be caused by adrenergic failure and result in profound orthostatic changes.

While transient drops in blood pressure may be left untreated, persistent hypertension should trigger action. Patient may be initially placed in Trendelenburg's position. Intravenous fluids and colloids may be used for volume expansion especially if there is suspicion that the patient may be hypovolemic. Vasoactive drugs should be used with caution because of the possibility of exaggerated response secondary to underlying denervation hypersensitivity. In our experience, most episodes of hypertension in patients with GBS can be successfully controlled with fluid administration. Sustained elevations of blood pressure of sufficient severity to warrant treatment are fortunately uncommon. In elderly patients, the threshold to treat hypertension should be lower in order to prevent complications from possible congestive heart failure. Beta blockers should be administered with great caution in patients with GBS since their use may be complicated by sudden hypotension and bradycardia. It is always preferable to use first agents with short half life and avoid very potent medications except in cases of true emergencies. Low doses of hydralazine or ACE inhibitors can be used in less severe cases. Very careful use of nicardipine or nitroprusside is reserved for very severe hypertension or cases with target organ complications.

Cardiac arrhythmias are the most threatening manifestation of autonomic dysfunction in patients with GBS [41]. Sudden bradycardia and asystole can result from exaggerated vagal activity. Tachyarrhythmias can originate from supraventricular or ventricular sources. Their treatment does not differ from arrhythmias produced by other causes. Reduced variation in the R-R interval and severe hypertension are predictors of increased risk for serious arrhythmias [43]. Whenever severe arrhythmia occurs, while dysautonomia 
may be the may be the most likely mechanism, other causes should be excluded, including myocardial ischemia, pulmonary embolism, or hypoxia. After an episode of symptomatic bradyarrhythmia, the risk of subsequent severe bradycardia and asystole is increased; therefore, these patients should be considered candidates for elective pacemaker placement unless a trigger for the bradycardia can be identified and avoided.

Other common manifestations of autonomic dysregulation that require attention and potential treatment include anhidrosis or excessive diaphoresis, urinary retention or most likely incontinence, gastroparesis, adynamic ileus, and constipation or less likely diarrhea. Bladder dysfunction is best treated with intermittent bladder catheterization. Stool softeners should be given routinely to all patients with GBS. Gastroparesis and ileus should be treated with intermittent nasogastric suctioning if necessary. Intravenous metoclopramide, a stimulant of gastric peristalsis, should be used with extreme caution because it has been reported to induce sinus arrest [44]. Adynamic ileus may occur in up to $15 \%$ of patients with severe GBS [30]. Its occurrence may be triggered by the use of opiates for pain. Combined use of gastric suctioning and rectal tube usually suffices to allow recovery of intestinal motility without complications. Although neostigmine has been used successfully in other patients with adynamic ileus, its use is much riskier in patients with GBS since it can be complicated by severe bradycardia. In refractory cases of ileus, parenteral nutrition may be needed to sustain the patient's caloric requirements.

\section{CONTROL OF PAIN}

Pain is common and frequently severe in patients with GBS [45]. It may be ameliorated by frequent body repositioning, physical therapy with stretching exercises, and use of analgesic medications when necessary. Acetaminophen and non-steroidal anti-inflammatory drugs such as ketorolac ( 15 to $30 \mathrm{mg}$ every six hours) are the usual first pharmacological choice. Tramadol 50 to $100 \mathrm{mg}$ every six hours represents another good option. Narcotics such as fentanyl or morphine should be reserved for cases with more intense or refractory pain. If opiates are prescribed, the patient should be particularly monitored for the high risk of developing ileus. Frequently, the pain has neuropathic features such as burning, tingling, or electric sensation. In these cases, the use of agents for neuropathic pain may be very beneficial. Options include tricyclic antidepressants (such as nortriptyline or amitriptyline), gabapentin, pregabalin, and duloxetine. When using tricyclic anti-depressants, it is important to keep in mind that these agents may result in exacerbation of orthostatic hypotension. While antidepressants may confer the added benefit of mood enhancement, this potential benefit does not become manifest until two to three weeks following initiation of the drug.

\section{NUTRITION}

Patients with GBS in the ICU have considerably increased metabolic requirements. This should be taken into account when calculating the patient's caloric needs. Enteral feeding should be instituted as soon as possible in patients with bulbar weakness or endotracheal intubation. The position of the tip of the feeding tube should be carefully checked before starting enteral feedings because patients with GBS have increased risk for aspiration. For this reason, radiographic confirmation of proper tube positioning is required in all cases. Enteral feeding should be started at low rates since patients with GBS often have gastroparesis which may result in high residual volumes. Subsequently, the rate of administration should be increased slowly to reach the target caloric supply. Patients must be frequently monitored for signs of ileus. Nutrition should be complemented with adequate intravenous hydration and increased insensible losses need to be taken into account in patients with profuse diaphoresis or diarrhea. Electrolytes should be monitored, especially sodium since patients with GBS may develop hyponatremia from inadequate secretion of antidiuretic hormone [46]. Parenteral nutrition should be reserved for patients with refractory ileus.

\section{GENERAL CARE MEASURES AND PREVENTION OF SYSTEMIC COMPLICATIONS}

Since patients with severe GBS may be immobilized for days, it is important to institute effective measures to prevent deep venous thrombosis [45]. Subcutaneous heparin (5000 units every 8 to 12 hours) should be combined with compression stockings and intermittent air compression devices. Patients who develop acute dyspnea and hypoxia should raise the suspicion of pulmonary embolism and prompt emergent investigations to exclude this diagnosis. When in doubt, intravenous heparin may be used until a pulmonary embolism can be excluded.

Stress gastroduodenal ulcers can be prevented by using $\mathrm{H} 2$ receptor blockers such as ranitidine or famotidine (proton-pump inhibitors such as pantoprazole can also be used but their efficacy for the prevention of stress ulcers has not been formally established). Frequent body turning, preferably every two hours while the patient is awake and every six hours during sleep, is necessary to prevent pressure sores and compression nerve palsies. Areas of particular risk for compression include the elbows and lateral aspects of the legs at the level of the fibula head regions (ulnar and peroneal nerve compression respectively). Using elbow pads and cushions between the legs when the patient is in lateral decubitus are useful interventions. Physical therapy should be started early since passive motion exercises are useful to prevent contractures, ameliorate venous stasis, and contribute to the patient's feeling of well-being.

Severe GBS can be a very unsettling experience for the patient. Psychological support should always be provided. Refractory cases with slow recovery may benefit from antidepressant medication.

\section{IMMUNOTHERAPY}

Immunomodulatory therapy has become the standard of care for the treatment of patients with severe GBS [47]. The options include plasma exchange and intravenous immunoglobulin. Corticosteroids have been repeatedly tested but, when used in isolation, they do not appear to confer any benefit. New immune treatment options are being investigated for the most refractory GBS cases. 


\section{PLASMA EXCHANGE}

Plasma exchange has been considered the gold standard for the immune treatment of patients with GBS for the last two decades. A Cochrane systematic review evaluated six trials comparing plasma exchange versus supportive care [48]. The benefit of plasma exchange was documented in a variety of outcome measures including improvement in disability grade, time to recovery of ambulation without aid, percentage of patients requiring artificial ventilation after four weeks, duration of mechanical ventilation, and full recovery of muscle strength after one year. Specifically, in five trials evaluating 623 participants, plasma exchange substantially reduced a proportion of patients requiring mechanical ventilation after four weeks (from $27 \%$ to $14 \%$; relative risk 0.53 ) [48]. In four studies evaluating this outcome, the recovery of full muscle strength after one year was increased from $55 \%$ in control subjects to $68 \%$ in patients treated with plasma exchange (relative risk 1.24). The benefits of plasma exchange have been documented for patients with severe GBS treated within four weeks of symptom onset and for patients with moderate disease (still ambulatory) when treated within two weeks of symptom onset. The benefit is greatest when that treatment is started earlier and there are no studies addressing the effect of plasma exchange when started more than 30 days after symptom onset.

The regimen of plasma exchange typically consists of exchanging one to two plasma volumes $(50-100 \mathrm{ml} / \mathrm{kg}$ ) on five separate occasions over one to two weeks. Single studies have shown that four plasma exchanges were superior to two, but six plasma exchanges are not superior to four. It is not clear whether continuous flow plasma exchange is superior to intermittent flow (one study showed superiority and another showed no significant difference between the two techniques). The preferred replacement fluid is albumin since a higher rate of adverse events was seen when fresh frozen plasma was used [49].

The rate of complications with plasma exchange in patients with GBS is relatively low. In fact, in the trials included in the Cochrane systematic review there were fewer cases of infectious complications and cardiac arrhythmias among patients treated with plasma exchange than in the control group [48]. Most complications are related to problems with venous access. The other potential adverse events include hypotension, septicemia, pneumonia, abnormal clotting, and hypocalcaemia. Although expensive, the cost of plasma exchange is more than offset by the savings in health care cost that result from shorter ICU and hospital stays [50].

In summary, plasma exchange is recommended for nonambulatory GBS patients within four weeks of symptom onset and for ambulatory GBS patients within two weeks of symptom onset [47].

\section{INTRAVENOUS IMMUNOGLOBULIN}

Since intravenous immunoglobulin came into use after plasma exchange had already become a proven treatment for patients with GBS, there have been no large trials comparing intravenous immunoglobulin with placebo for this indication. In a small trial comparing intravenous immunoglobulin with supportive treatment in a population of children showed that recovery was hastened by intravenous immunoglobulin
[51]. There have been six randomized trials comparing intravenous immunoglobulin with plasma exchange. These trials were systematically analyzed in a Cochrane systematic review [52]. Most participants in these trials were unable to walk unaided and had developed their first symptoms less than two weeks before enrollment. There were no significant differences between plasma exchange and intravenous immunoglobulin in these trials in various outcome measures including improvement in disability after four weeks, duration of mechanical ventilation, recovery of an unaided ambulation, and proportion of patients dead or disabled after one year. A small study in children suggested that intravenous immunoglobulin could hasten recovery compared with plasma exchange; however, this differential effect remains to be confirmed [53].

In general, the rate of adverse events in these trials was lower among patients treated with intravenous immunoglobulin [52]. Particularly, multiple complications were more common with plasma exchange [54]. However, the lack of standardized definitions for side effects across the studies prevents a meta-analysis using the occurrence of complications as endpoint. Overall, both therapies are relatively safe and the choice of which one to use depends primarily on the experience of each particular center. The most common adverse events with intravenous immunoglobulin are vomiting, meningismus, renal failure, myocardial infarction and infusion site erythema.

The regimen of administration of intravenous immunoglobulin is typically $0.4 \mathrm{~g} / \mathrm{kg}$ per day for five days. The mechanism of action of intravenous immunoglobulin is not fully clarified and probably complex and multifactorial. It may involve blockade of $\mathrm{Fc}$ receptors, provision of antiidiotypic antibodies, interference with complement activation, and T-cell regulation [55].

In conclusion, intravenous immunoglobulin is recommended for patients with severe GBS who present within two weeks of symptom onset [47]. Although, intravenous immunoglobulin has not been formally evaluated when started after two weeks from symptom onset or given to patients with moderate disability, it may be a reasonable alternative to plasma exchange in these patients as well.

\section{COMBINATION TREATMENT}

Only one trial has compared the combined strategy of plasma exchange followed by intravenous immunoglobulin against plasma exchange alone [56]. The results showed no significant benefit from the combination regimen. There were more complications in the patients enrolled to the combination treatment group. There was also no difference in any outcome measure between patients treated with plasma exchange followed by intravenous immunoglobulin and those treated with intravenous immunoglobulin alone. Still, the sample size of this study was not sufficiently large to exclude a small beneficial effect from combination therapy [53].

Intravenous immunoglobulin given after immunoabsorption was not superior to immunoabsorption alone in another smaller study. Therefore, sequential treatment with plasma exchange followed by intravenous immunoglobulin is not recommended based on current evidence [47]. Sequential use 
of intravenous immunoglobulin followed by plasma exchange has not been tested, but this strategy appears counterintuitive because plasma exchange would be "washing out" the immunoglobulin recently administered.

A problem that remains unsolved is how to manage patients who show no signs of recovery two weeks or more after treatment with plasma exchange or intravenous immunoglobulin. Very preliminary evidence has suggested that a second course of intravenous immunoglobulin in these refractory cases might be beneficial but more studies exclusively focusing on these recalcitrant cases are needed.

\section{CORTICOSTEROIDS}

Six randomized trials testing corticosteroids in patients with GBS were analyzed by a Cochrane systematic review [57]. No significant difference between the corticosteroid and control groups was noted in disability grade four weeks after symptom onset. In addition, there were no differences in a number of secondary outcome measures including time to recovery of unaided walking, time to discontinuation of mechanical ventilation, mortality, and combined mortality and disability after one year. Various forms of steroids were tested in these trials including ACTH, intravenous methylprednisolone, oral prednisolone, and prednisone.

Complications were similar in the corticosteroid and placebo groups and no detrimental effect on muscle strength was noted in corticosteroid-treated patients. This lack of adverse effects supported the design of a trial which compared intravenous methylprednisolone (500 $\mathrm{mg}$ daily for five days) in addition to intravenous immunoglobulin against intravenous immunoglobulin alone [58]. The addition of steroids in this trial resulted in a non-significant trend toward faster improvement, although the effect only became significant in the post-hoc analysis after correction for prognostic factors including age and initial disability.

Given the heterogeneity across trials, studies testing oral and intravenous corticosteroids have been grouped separately. In the four small trials which used oral corticosteroids (total 120 participants), there was significantly less improvement in patients treated with corticosteroids versus those who were not [53]. Meanwhile, the two larger trials employing intravenous methlyprednisolone (total 467 participants) indicated greater improvement in the corticosteroid-treated patients, although the difference did not reach statistical significance [53,58,59].

There has been considerable debate regarding the possible reasons that might explain the lack of clear benefit of corticosteroid therapy in patients with GBS. It has been postulated that corticosteroids could inhibit macrophage clearance of myelinating debris, thus hampering attempts to remyelination or aggravating damage of denervated muscle fibers [60].

In summary, corticosteroids are not recommended for the treatment of patients with GBS [47]. There may be a role for high-dose intravenous corticosteroids administered to the most severe cases with GBS in addition to intravenous immunoglobulin or even potentially to plasma exchange, but this indication needs to be explored further in future studies.

\section{OTHER TESTED IMMUNE TREATMENTS AND FU- TURE OPTIONS}

Immunoabsorption and CSF filtration do not appear to offer any advantage over plasma exchange or intravenous immunoglobulin therapies. The increased complexity of their implementation is therefore not justified. Innovative treatments which deserve to be tested in the future include treatments designed to modulate T-cell function (such as interferons), sodium channel blockers that may protect axons against injury, and neurotrophic factors that may ameliorate nerve damage and accelerate reinnervation.

\section{PROGNOSIS}

Although patients with GBS typically survive their acute disease and improve over time, persistent symptoms are common and long-term disability occurs in up to $20 \%$ of cases. In a multicenter perspective study of 297 patients with GBS with an average follow-up of 309 days, it was observed that $71 \%$ of patients recovered favorably, $16 \%$ had residual deficits, and $11 \%$ died [61]. In this population, the mean time to nadir was 12 days, to improvement 28 days, and to clinical recovery 200 days. As expected, symptom progression had plateaued by four weeks in $98 \%$ of the patients. Improvement had started within four weeks of symptom onset in $85 \%$. Chances of recovery were negatively affected by older age, preceding gastroenteritis, preexistent disability, electrophysiological evidence of axonal damage, longer time to clinical nadir, and more prolonged duration of the acute disease. Although in this study, the treatments used did not appear to influence the likelihood of long-term recovery, previous trials demonstrated that treatment with plasma exchange or intravenous immunoglobulin increases the chances of successful functional recovery at one year.

Among patients with GBS who require mechanical ventilation, the mortality is $20 \%$, but most survivors achieve meaningful functional recovery including independent ambulation (Fig. 2) [62]. In fact, young patients may recover normal function despite prolonged ventilation. Older age and delayed transfer to a tertiary center have been identified as independent predictors of poor prognosis among ventilated patients with GBS [62].

Fig. (2). Long-term outcome of ventilated patients with GuillainBarré syndrome. Reproduced with permission from Fletcher et al. [62]. 
Axonal damage is widely regarded as a marker of poor functional prognosis. However, electrophysiological evidence of axonal neuropathy may carry a better prognosis than previously assumed. In a Japanese study of 44 consecutive patients with the severe acute motor axonal neuropathy form of GBS, only six of the patients (14\%) were unable to walk independently at six months and four of them had regained independent ambulation after one year [63]. Therefore, it is becoming increasingly evident that while patients with axonal GBS progress faster to nadir during the acute phase of the disease, their functional recovery may be as complete as in patients with demyelinating GBS.

A mild degree of residual neuropathy effecting large and medium sized myelinated fibers may occur in close to half of all patients with GBS [64]. This neuropathy often has a clinical correlate in the form of sensory and motor symptoms that, although not disabling, may worsen quality of life. In addition, persistent symptoms of autonomic dysfunction are not infrequent, including orthostatic hypotension, impotence, and bladder dysfunction. These residual signs and symptoms are more common in older patients. Because of these symptoms, especially fatigue, close to one-third of patients who have experienced an episode of GBS are forced to make substantial changes in their daily lives even after nearly full recovery [65].

\section{REFERENCES}

[1] Guillain G, Barré JA, Strohl A. Sur en syndrome de radiculonevrite avec hyperalbuminose du liquide cephalo-rachiden sans reaction cellulaire: Remarques sur les caracteres cliniques et graphiques der reflexes tendineaux. Bull Soc Med Hosp Paris 1916; 40: 14621470.

[2] Hughes RA, Cornblath DR. Guillain-Barre syndrome. Lancet 2005; 366(9497): 1653-1666.

[3] Hughes RA, Rees JH. Clinical and epidemiologic features of Guillain-Barre syndrome. J Infect Dis 1997; 176 Suppl 2: S92-8.: S92S98.

[4] Govoni V, Granieri E. Epidemiology of the Guillain-Barre syndrome. Curr Opin Neurol 2001; 14(5): 605-613.

[5] Ho TW, Mishu B, Li CY, Gao CY, Cornblath DR, Griffin JW, et al. Guillain-Barre syndrome in northern China. Relationship to Campylobacter jejuni infection and anti-glycolipid antibodies. Brain 1995; 118(Pt 3): 597-605.

[6] van Koningsveld R, Rico R, Gerstenbluth I, Schmitz PI, Ang CW, Merkies IS, et al. Gastroenteritis-associated Guillain-Barre syndrome on the Caribbean island Curacao. Neurology 2001; 56(11): 1467-1472.

[7] McKhann GM, Cornblath DR, Ho T, Li CY, Bai AY, Wu HS, et al. Clinical and electrophysiological aspects of acute paralytic disease of children and young adults in northern China. Lancet 1991; 338(8767): 593-597.

[8] Winer JB, Hughes RA, Anderson MJ, Jones DM, Kangro H, Watkins RP. A prospective study of acute idiopathic neuropathy. II. Antecedent events. J Neurol Neurosurg Psychiatry 1988; 51(5): 613-618.

[9] Hadden RD, Karch H, Hartung HP, Zielasek J, Weissbrich B, Schubert J, et al. Preceding infections, immune factors, and outcome in Guillain-Barre syndrome. Neurology 2001; 56(6): 758765 .

[10] Haber P, DeStefano F, Angulo FJ, Iskander J, Shadomy SV, Weintraub E, et al. Guillain-Barre syndrome following influenza vaccination. JAMA 2004; 292(20): 2478-2481.

[11] Brannagan TH, III, Zhou Y. HIV-associated Guillain-Barre syndrome. J Neurol Sci 2003; 208(1-2): 39-42.

[12] Gabriel CM, Gregson NA, Redford EJ, Davies M, Smith KJ, Hughes RA. Human immunoglobulin ameliorates rat experimental autoimmune neuritis. Brain 1997; 120(Pt 9): 1533-1540.

[13] Prineas JW. Pathology of the Guillain-Barre syndrome. Ann Neurol 1981; 9 Suppl: 6-19.
[14] Hafer-Macko CE, Sheikh KA, Li CY, Ho TW, Cornblath DR, McKhann GM, et al. Immune attack on the Schwann cell surface in acute inflammatory demyelinating polyneuropathy. Ann Neurol 1996; 39(5): 625-635.

[15] Hafer-Macko C, Hsieh ST, Li CY, Ho TW, Sheikh K, Cornblath DR, et al. Acute motor axonal neuropathy: an antibody-mediated attack on axolemma. Ann Neurol 1996; 40(4): 635-644.

[16] Illa I, Ortiz N, Gallard E, Juarez C, Grau JM, Dalakas MC. Acute axonal Guillain-Barre syndrome with IgG antibodies against motor axons following parenteral gangliosides. Ann Neurol 1995; 38(2): 218-224.

[17] Willison HJ, Yuki N. Peripheral neuropathies and anti-glycolipid antibodies. Brain 2002; 125(Pt 12): 2591-2625.

[18] Ogawara K, Kuwabara S, Koga M, Mori M, Yuki N, Hattori T. Anti-GM1b IgG antibody is associated with acute motor axonal neuropathy and Campylobacter jejuni infection. J Neurol Sci 2003; 210(1-2): 41-45.

[19] Sheikh KA, Ho TW, Nachamkin I, Li CY, Cornblath DR, Asbury $\mathrm{AK}$, et al. Molecular mimicry in Guillain-Barre syndrome. Ann N Y Acad Sci 1998; \%19; 845: 307-21.: 307-321.

[20] Yuki N. Ganglioside mimicry and peripheral nerve disease. Muscle Nerve 2007; 35(6): 691-711.

[21] Koga M, Gilbert M, Takahashi M, Li J, Koike S, Hirata K, et al. Comprehensive analysis of bacterial risk factors for the development of Guillain-Barre syndrome after Campylobacter jejuni enteritis. J Infect Dis 2006; 193(4): 547-555.

[22] Perera VN, Nachamkin I, Ung H, Patterson JH, McConville MJ, Coloe PJ, et al. Molecular mimicry in Campylobacter jejuni: role of the lipo-oligosaccharide core oligosaccharide in inducing antiganglioside antibodies. FEMS Immunol Med Microbiol 2007; 50(1): 27-36.

[23] Yuki N, Susuki K, Koga M, Nishimoto Y, Odaka M, Hirata K, et al. Carbohydrate mimicry between human ganglioside GM1 and Campylobacter jejuni lipooligosaccharide causes Guillain-Barre syndrome. Proc Natl Acad Sci USA 2004; 101(31): 11404-11409.

[24] Kuwabara S, Ogawara K, Misawa S, Koga M, Mori M, Hiraga A, et al. Does Campylobacter jejuni infection elicit "demyelinating" Guillain-Barre syndrome? Neurology 2004; 63(3): 529-533.

[25] Kuwabara S, Ogawara K, Koga M, Mori M, Hattori T, Yuki N. Hyperreflexia in Guillain-Barre syndrome: relation with acute motor axonal neuropathy and anti-GM1 antibody. J Neurol Neurosurg Psychiatry 1999; 67(2): 180-184.

[26] Asbury AK, Cornblath DR. Assessment of current diagnostic criteria for Guillain-Barre syndrome. Ann Neurol 1990; 27 Suppl: S214.: S21-S24.

[27] Fulgham JR, Wijdicks EF. Guillain-Barre syndrome. Crit Care Clin 1997; 13(1): 1-15.

[28] Ropper AH, Wijdicks EF. Blood pressure fluctuations in the dysautonomia of Guillain-Barre syndrome. Arch Neurol 1990; 47(6): 706-708.

[29] Pfeiffer G, Schiller B, Kruse J, Netzer J. Indicators of dysautonomia in severe Guillain-Barre syndrome. J Neurol 1999; 246(11): 1015-1022.

[30] Burns TM, Lawn ND, Low PA, Camilleri M, Wijdicks EF. Adynamic ileus in severe Guillain-Barre syndrome. Muscle Nerve 2001; 24(7): 963-965.

[31] Bernstein R, Mayer SA, Magnano A. Neurogenic stunned myocardium in Guillain-Barre syndrome. Neurology 2000; 54(3): 759762.

[32] Ropper AH, Wijdicks EF, Shahani BT. Electrodiagnostic abnormalities in 113 consecutive patients with Guillain-Barre syndrome. Arch Neurol 1990; 47(8): 881-887.

[33] Orlikowski D, Prigent H, Sharshar T, Lofaso F, Raphael JC. Respiratory dysfunction in Guillain-Barre Syndrome. Neurocrit Care 2004; 1(4): 415-422.

[34] Rabinstein AA, Wijdicks EF. Warning signs of imminent respiratory failure in neurological patients. Semin Neurol 2003; 23(1): 97 104.

[35] Lawn ND, Fletcher DD, Henderson RD, Wolter TD, Wijdicks EF. Anticipating mechanical ventilation in Guillain-Barre syndrome. Arch Neurol 2001; 58(6): 893-898.

[36] Wijdicks EF, Henderson RD, McClelland RL. Emergency intubation for respiratory failure in Guillain-Barre syndrome. Arch Neurol 2003; 60(7): 947-948.

[37] Wijdicks EF, Roy TK. BiPAP in early guillain-barre syndrome may fail. Can J Neurol Sci 2006; 33(1): 105-106. 
[38] Lawn ND, Wijdicks EF. Post-intubation pulmonary function test in Guillain-Barre syndrome. Muscle Nerve 2000; 23(4): 613-616.

[39] Lawn ND, Wijdicks EF. Tracheostomy in Guillain-Barre syndrome. Muscle Nerve 1999; 22(8): 1058-1062.

[40] Henderson RD, Lawn ND, Fletcher DD, McClelland RL, Wijdicks EF. The morbidity of Guillain-Barre syndrome admitted to the intensive care unit. Neurology 2003;60(1): 17-21.

[41] Greenland P, Griggs RC. Arrhythmic complications in the GuillainBarre syndrome. Arch Intern Med 1980; 140(8): 1053-1055.

[42] Narayan D, Huang MT, Mathew PK. Bradycardia and asystole requiring permanent pacemaker in Guillain-Barre syndrome. Am Heart J 1984; 108(2): 426-428.

[43] Winer JB, Hughes RA. Identification of patients at risk of arrhythmia in the Guillain-Barre syndrome. Q J Med 1988; 68(257): 735739.

[44] Malkoff MD, Ponzillo JJ, Myles GL, Gomez CR, Cruz-Flores S. Sinus arrest after administration of intravenous metoclopramide. Ann Pharmacother 1995; 29(4): 381-383.

[45] Hughes RA, Wijdicks EF, Benson E, Cornblath DR, Hahn AF, Meythaler JM, et al. Supportive care for patients with guillainbarre syndrome. Arch Neurol 2005; 62(8): 1194-1198.

[46] Cooke CR, Latif KA, Huch KM, Wall BM. Inappropriate antidiuresis and hyponatremia with suppressible vasopressin in GuillainBarre syndrome. Am J Nephrol 1998; 18(1): 71-76.

[47] Hughes RA, Wijdicks EF, Barohn R, Benson E, Cornblath DR, Hahn AF, et al. Practice parameter: immunotherapy for GuillainBarre syndrome: report of the Quality Standards Subcommittee of the American Academy of Neurology. Neurology 2003; 61(6): 736-740.

[48] Raphael JC, Chevret S, Hughes RA, Annane D. Plasma exchange for Guillain-Barre syndrome. Cochrane Database Syst Rev 2001; (2): CD001798.

[49] French Cooperative Group on Plasma Exchange in Guillain-Barre syndrome. Efficiency of plasma exchange in Guillain-Barre syndrome: role of replacement fluids. Ann Neurol 1987; 22(6): 753761

[50] Osterman PO, Fagius J, Lundemo G, Pihlstedt P, Pirskanen R, Siden A, et al. Beneficial effects of plasma exchange in acute inflammatory polyradiculoneuropathy. Lancet 1984; 2(8415): 12961299.

[51] Gurses N, Uysal S, Cetinkaya F, Islek I, Kalayci AG. Intravenous immunoglobulin treatment in children with Guillain-Barre syndrome. Scand J Infect Dis 1995; 27(3): 241-243.

[52] Hughes RA, Raphael JC, Swan AV, van Doorn PA. Intravenous immunoglobulin for Guillain-Barre syndrome. Cochrane Database Syst Rev 2006; (1): CD002063.
[53] Hughes RA, Swan AV, Raphael JC, Annane D, van Koningsveld R, van Doorn PA. Immunotherapy for Guillain-Barre syndrome: a systematic review. Brain 2007.

[54] van der Meche FG, Schmitz PI. A randomized trial comparing intravenous immune globulin and plasma exchange in GuillainBarre syndrome. Dutch Guillain-Barre Study Group. N Engl J Med 1992; 326(17): 1123-1129.

[55] Dalakas MC. Intravenous immunoglobulin in autoimmune neuromuscular diseases. JAMA 2004; 291(19): 2367-2375.

[56] Plasma Exchange/Sandoglobulin Guillain-Barre Syndrome Trial Group. Randomised trial of plasma exchange, intravenous immunoglobulin, and combined treatments in Guillain-Barre syndrome. Lancet 1997; 349(9047): 225-230.

[57] Hughes RA, Swan AV, van Koningsveld R, van Doorn PA. Corticosteroids for Guillain-Barre syndrome. Cochrane Database Syst Rev 2006; 19(2): CD001446.

[58] van Koningsveld R, Schmitz PI, Meche FG, Visser LH, Meulstee J, van Doorn PA. Effect of methylprednisolone when added to standard treatment with intravenous immunoglobulin for Guillain-Barre syndrome: randomised trial. Lancet 2004; 363(9404): 192-196.

[59] Double-blind trial of intravenous methylprednisolone in GuillainBarre syndrome. Guillain-Barre Syndrome Steroid Trial Group. Lancet 1993; 341(8845): 586-590.

[60] Rich MM, Pinter MJ. Sodium channel inactivation in an animal model of acute quadriplegic myopathy. Ann Neurol 2001; 50(1) 26-33.

[61] The Italian Guillain-Barre Study Group. The prognosis and main prognostic indicators of Guillain-Barre syndrome. A multicentre prospective study of 297 patients. Brain 1996; 119(Pt 6): 20532061.

[62] Fletcher DD, Lawn ND, Wolter TD, Wijdicks EF. Long-term outcome in patients with Guillain-Barre syndrome requiring mechanical ventilation. Neurology 2000; 54(12): 2311-2315.

[63] Hiraga A, Mori M, Ogawara K, Kojima S, Kanesaka T, Misawa S, et al. Recovery patterns and long term prognosis for axonal Guillain-Barre syndrome. J Neurol Neurosurg Psychiatry 2005; 76(5): 719-722.

[64] Dornonville dlC, Jakobsen J. Residual neuropathy in long-term population-based follow-up of Guillain-Barre syndrome. Neurology 2005; 64(2): 246-253.

[65] Bersano A, Carpo M, Allaria S, Franciotta D, Citterio A, NobileOrazio E. Long term disability and social status change after Guillain-Barre syndrome. J Neurol 2006; 253(2): 214-218. 